\title{
A judicialização de medicamentos imunoterápicos sem registro na Anvisa: o caso do Estado de São Paulo
}

The judicialization of immunotherapy drugs without registration with Anvisa: the case of the State of São Paulo, Brazil

La judicialización de los medicamentos de inmunoterapia sin registro en Anvisa: el caso del Estado de São Paulo, Brasil

Daniel Buffone de Oliveira ${ }^{1}$

Rafael Augusto Mantovani Silva²

Ellen Alves de Paula ${ }^{3}$

Raphael Pereira Cassamassimo 4

Paula Sue Facundo de Siqueira ${ }^{5}$

Daniela Oliveira de $\mathrm{Melo}^{6}$

\section{Resumo}

Objetivos: o registro sanitário garante minimamente a segurança nas indicações de bula. Com o julgamento do Recurso Extraordinário (RE) № 657.718/2019, essa questão não está pacificada. $\mathrm{O}$ objetivo desse estudo é comparar as indicações aprovadas nas agências sanitárias americana, europeia e brasileira e analisar dados referentes à indicação de medicamentos imunoterápicos para o tratamento de câncer, em demandas judiciais contra a Secretaria de Estado da Saúde de São Paulo (SES/SP). Métodos: foi realizada uma pesquisa documental nas bulas de seis medicamentos imunoterápicos, disponíveis em julho de 2019 nos websites das agências sanitárias elegidas para o estudo, e comparada a indicação desses medicamentos em demandas judiciais no Estado de São Paulo, utilizando os dados de relatórios ou documentos escaneados disponíveis no sistema S-Codes. Resultados: todos os medicamentos têm registro sanitário para, ao menos, uma indicação em bula nas três agências sanitárias, porém, com diferenças nas indicações aprovadas, muitas delas sendo aprovações aceleradas (fast track). $O$ tempo médio entre a aprovação na Food and Drug Administration (FDA) e na Agência de Vigilância Sanitária (Anvisa) foi de 464,5 \pm 170,8 dias; e 278 (98\%) das demandas judiciais ocorreram pós-registro na Anvisa. Há pouca informação disponível nos documentos escaneados, mas foi possível identificar

\footnotetext{
${ }^{1}$ Farmacêutico; diretor técnico II da Secretaria Estadual de Saúde do Estado de São Paulo, São Paulo, SP, Brasil. E-mail: dbuffone@saude.sp.gov.br

2 Farmacêutico; colaborador do Núcleo de Avaliação de Tecnologias em Saúde (NATS) da Unifesp, Diadema, SP. E-mail: rafaa.mantovani@gmail.com

3 Especialista em ATS; farmacêutica; colaboradora do Núcleo de Avaliação de Tecnologias em Saúde (NATS) da Unifesp, Diadema, SP, Brasil. E-mail: 13ellen@gmail.com

4 Graduando em Farmácia pela Universidade Faculdades Metropolitanas Unidas; estagiário do Grupo de Coordenação das Demandas Estratégicas do SUS (GCODES), São Paulo, SP, Brasil. E-mail: raphaelcassamassimo.farmacia@gmail.com 5 Enfermeira; coordenadora do Grupo de Coordenação das Demandas Estratégicas do SUS (GCODES), São Paulo, SP, Brasil. E-mail: psue@saude.sp.gov.br

6 Doutora; docente adjunta do Instituto de Ciências Ambientais, Químicas e Farmacêuticas e coordenadora do Núcleo de Avaliação de Tecnologias em Saúde (NATS) da Unifesp, Diadema, SP, Brasil. E-mail: melo.daniela@unifesp.br
} 
situações envolvendo indicações de medicamentos, além de resultados de testes genéticos. Discussão e conclusão: a análise mostra que a FDA tende a ser menos rigorosa na aprovação de novas indicações, e que a maioria das demandas não se enquadraria nos critérios do RE no 657.718/2019. Apesar do avanço, faz-se necessária a discussão do uso off-label desses medicamentos e sua especificidade.

Palavras-chave: Judicialização da saúde. Uso off-label. Assistência farmacêutica. Acesso a medicamentos essenciais e tecnologias em saúde. Agência Nacional de Vigilância Sanitária.

\begin{abstract}
Objectives: the sanitary registry minimally guarantees the safety of the labeling instructions. With the judgment of Extraordinary Appeal (RE) No. 657,718/2019, this issue is not pacified. The aim of this study is to compare the indications approved by the American, European and Brazilian health agencies and to analyze data regarding the indication of immunotherapy drugs for cancer treatment, in lawsuits against the São Paulo State Department of Health (SES / SP). Methods: a documentary research was performed on the package leaflets of six immunotherapeutic drugs, available in July 2019 on the websites of the health agencies eligible for the study, and comparing the indication of these drugs in lawsuits in the State of São Paulo, using data from reports or scanned documents available on the S-Codes system. Results: all drugs have a health record for at least one label indication in the three health agencies, but with differences in approved indications, many of them being fast track approvals. The median time between approval by the Food and Drug Administration (FDA) and the Health Surveillance Agency (Anvisa) was $464.5 \pm 170.8$ days; and $278(98 \%$ ) of the lawsuits occurred after registration with Anvisa. There is little information available in the scanned documents, but it was possible to identify situations involving drug indications as well as genetic test results. Discussion and conclusion: the analysis shows that the FDA tends to be less rigorous in approving new indications, and that most demands would not fit the criteria of RE 657.718/2019. Despite the progress, it is necessary to discuss the off-label use of these drugs and their specificity.
\end{abstract}

Keywords: Health's judicialization. Off-label use. Pharmaceutical services. Access to essential medicines and health technologies. Brazilian Health Surveillance Agency.

\title{
Resumen
}

Objetivos: el registro sanitario garantiza mínimamente la seguridad de las instrucciones de etiquetado. Con la sentencia de Apelación Extraordinaria (RE) No. 657,718 / 2019, este asunto no se pacifica. El objetivo de este estudio es comparar las indicaciones aprobadas por las agencias de salud estadounidenses, europeas y brasileñas y analizar datos sobre la indicación de medicamentos de inmunoterapia para el tratamiento del cáncer, en demandas contra el Departamento de Salud del Estado de São Paulo (SES / SP). Métodos: se realizó una investigación documental en los prospectos de seis medicamentos inmunoterapéuticos, disponibles en julio de 2019, en los sitios web de las agencias de salud elegibles para el estudio. documentos escaneados disponibles en el sistema S-Codes. Resultados: todos los medicamentos tienen un historial médico para al menos una indicación de etiqueta en las tres agencias de salud, pero con diferencias en las indicaciones aprobadas, muchas de ellas son aprobaciones aceleradas. El tiempo promedio entre la aprobación de la Food and Drug Administration (FDA) y la Agencia de Vigilancia de la Salud (Anvisa) fue de $464.5 \pm 170.8$ 
días; y 278 (98\%) de las demandas ocurrieron después de registrarse con Anvisa. Hay poca información disponible en los documentos escaneados, pero fue posible identificar situaciones que implican indicaciones de drogas, así como resultados de pruebas genéticas. Discusión y conclusión: el análisis muestra que la FDA tiende a ser menos rigurosa en la aprobación de nuevas indicaciones, y que la mayoría de las demandas no se ajustan a los criterios de RE 657.718/2019. A pesar del progreso, es necesario discutir el uso fuera de etiqueta de estos medicamentos y su especificidad.

Palabras-llave: Judicialización de la salud. Uso fuera de lo indicado. Servicios farmacéuticos. Acceso a medicamentos esenciales y tecnologías sanitarias. Agencia Nacional de Vigilancia Sanitaria.

\section{Introdução}

Em maio de 2019, o Supremo Tribunal Federal (STF) apreciou o Recurso Extraordinário (RE) no 657.718/2019 e julgou o mérito com repercussão geral; foi decidido que o Estado não poderia ser obrigado a fornecer medicamentos experimentais, ou seja, aqueles que não possuem aprovação junto à Agência Nacional de Vigilância Sanitária (Anvisa), ao menos em casos específicos. É importante destacar que a questão do registro sanitário não dizia respeito apenas às dificuldades em processos de importação de medicamentos, mas sim ao fornecimento de medicamentos pelo Sistema Único de Saúde (SUS) sem que tivesse sido comprovada sua eficácia e segurança junto à Agência sanitária brasileira, o que seria considerado tratamento experimental (1).

O registro sanitário de um medicamento permite sua comercialização para uma ou mais indicações para as quais tenham sido apresentados estudos clínicos pelo demandante. É comum a mudança de bula para a ampliação de indicação ou inclusão de novas indicações para um medicamento, mediante a apresentação de resultados de estudos clínicos pela indústria farmacêutica, o que reforça a ideia de que o registro considera o binômio medicamento/indicação (2-4). O uso de medicamentos para indicações que não constam em bula é conhecido como off-label e o RE n 657.718/2019 não abordou esse aspecto. Tratase uma realidade já bastante conhecida, principalmente entre pacientes pediátricos, devido à falta de opções terapêuticas. No Brasil, o caso recente mais famoso, inclusive no meio jurídico, envolveu o medicamento bevacizumabe - um medicamento com registro no Brasil, desde 2002, para o tratamento de carcinoma metastático. Em 2016, a Anvisa publicou a RDC n 111/2016, autorizando seu uso excepcional no tratamento da degeneração macular relacionada à idade, considerando a solicitação da Comissão Nacional de Incorporação de 
Tecnologias (Conitec) no SUS (5). O uso off-label de medicamentos na oncologia não é uma novidade. De 43 medicamentos oncológicos aprovados pelo órgão do governo dos Estados Unidos, Food and Drug Administration (FDA), entre 1999 e 2011, 34 (79\%) apresentavam pelo menos um uso off-label bem aceito na prática clínica, ou seja, amplamente empregado (6).

A questão do uso off-label pode representar um desafio ainda maior no caso dos medicamentos oncológicos, diante da gravidade do quadro clínico dos pacientes e o alto custo dos novos medicamentos. Além disso, sabe-se que uma proporção dos tumores é causada por mutações genéticas e/ou apresenta melhor ou pior resposta aos medicamentos, dependendo do perfil genético e/ou molecular. A implementação da oncologia de precisão, ou seja, o uso de terapia alvo com base no perfil molecular do tumor ou genético do paciente, tem sido amplamente discutida no mundo todo (7-9). A oncologia de precisão, ou medicina personalizada, foi pauta de recente audiência no Senado Federal, que teve como objetivo promover a discussão da importância do uso de medicamentos específicos para marcadores genéticos presentes nas células cancerígenas (10).

As mutações têm papel relevante na etiologia do câncer, sendo alterações na sequência de um ou mais genes (genótipo) que podem ou não resultar em alterações perceptíveis, de gravidade variável. O DNA possui locais em que as mutações ocorrem em frequência muito superior ao normal - até 100 vezes -, os chamados hot spots (11-13). Com o recente avanço na área de biologia molecular, tem sido possível a identificação de um número cada vez maior de mutações, sobretudo aquelas que ocorrem em hot spots; mas, apesar do avanço, esse conhecimento será traduzido em benefício para uma pequena proporção de pacientes, já que $85 \%$ das mutações estão presentes em menos de $5 \%$ do tipo tumoral em que são encontradas (11-14). Portanto, a oncologia de precisão permanece mais como uma promessa do que realidade, além de ter um custo muito alto e ser elegível apenas para uma pequena parcela dos pacientes (7-9).

Recentemente foram publicados dois estudos estimando a porcentagem de pacientes com câncer, nos Estados Unidos, que seriam elegíveis ao tratamento e/ou responderiam aos medicamentos representantes da terapia alvo - guiada pela identificação de mutações - e os imunoterápicos, medicamentos que inibem o controle imunológico, muitas vezes utilizado pelas células tumorais para evitarem serem reconhecidas e atacadas, para que o corpo do 
paciente passe a se defender do tumor $(15,16)$.

Ambos os grupos de medicamentos são considerados um enorme avanço tecnológico, com aumento das publicações a esse respeito e do investimento em seu desenvolvimento $(15,16)$. No entanto, poucos pacientes seriam elegíveis para o tratamento e menos ainda de fato responderiam ao tratamento, considerando-se as estimativas dos ensaios clínicos para todos os tumores para os quais têm indicação aprovada pela FDA tanto com terapia alvo (15,44\% seriam elegíveis e 4,9\% responderiam, em 2018) quanto com imunoterápicos (44\% elegíveis e 13\% responderiam a terapia em 2018) - em condições de acesso total a esses medicamentos $(15,16)$.

Os imunoterápicos têm sido alvo de amplo interesse devido à capacidade de gerar respostas duradouras em muitos tumores malignos, até então intratáveis, e promover melhorias na sobrevida global dos pacientes que respondem ao tratamento (16). Os maiores problemas são justamente o fato de que a parcela que responde a esses medicamentos é realmente muito pequena e, claro, o alto custo do tratamento. Entre os seis medicamentos imunoterápicos - atezolizumabe, avelumabe, durvalumabe, ipilimmumabe, nivolumabe e pembrolizumabe -, todos têm registro no Brasil para ao menos uma indicação; e dois deles estão entre os 30 medicamentos com os quais a Secretaria de Estado da Saúde de São Paulo (SES/SP) mais gastou em 2018: nivolumabe, na sétima posição; e pembrolizumabe; na 26a . O objetivo deste trabalho é comparar as bulas dos medicamentos imunoterápicos nos Estados Unidos (FDA), União Europeia (European Medicines Agency - EMA) e Brasil (Anvisa) para identificar diferenças e analisar dados referentes à indicação do medicamento em demandas judiciais contra a SES/SP para obtenção desses medicamentos.

\section{Métodos}

Comparação de bulas e tempo de aprovação do registro

As bulas dos medicamentos atezolizumabe, avelumabe, durvalumabe, ipilimmumabe, nivolumabe e pembrolizumabe, foram consultadas nos websites das agências sanitárias FDA, EMA e Anvisa, sendo as indicações extraídas e compiladas de forma a facilitar a comparação. O texto das bulas da FDA e EMA foi traduzido para o português. Tendo em vista a dinâmica de aprovação de novas indicações na categoria de medicamentos oncológicos, essa análise foi conduzida utilizando as versões das bulas disponíveis em julho 
de 2019, nas três agências reguladoras. Também foi coletada a data do registro da primeira(s) indicação(ões) em cada agência sanitária para o cálculo do tempo, em dias, entre o registro na FDA, na EMA e na Anvisa.

\section{Análise das demandas judiciais}

Foi realizada uma consulta no sistema S-Codes, em 15 de julho de 2019, por informações sobre demandas judiciais envolvendo os medicamentos elencados no estudo, por meio de relatório gerado pelo sistema, filtrando-se pelos medicamentos atezolizumabe, avelumabe, durvalumabe, ipilimmumabe, nivolumabe ou pembrolizumabe.

O S-Codes é um sistema de informação planejado e desenvolvido pela Secretaria de Estado de Saúde de São Paulo (SES/SP), implantado integralmente em todo o Estado em 2010. A partir do cadastro das demandas judiciais de todos os Departamentos Regionais de Saúde (DRS), divisões administrativas da SES/SP, permite o controle das demandas judiciais ajuizadas contra o órgão.

$\mathrm{Na}$ inclusão da demanda no sistema S-Codes, há um campo para a indicação do Código Internacional de Doenças (CID 10), porém não há como verificar detalhamentos como estadiamento do tumor, presença ou não de metástases ou tratamentos prévios em relatórios do sistema. O CID é incluído pelo operador com base no CID do laudo médico que consta no processo ou pela interpretação das informações do laudo - quando o CID não foi descrito pelo prescritor do medicamento. Foi realizada uma análise das indicações para as quais os medicamentos foram solicitados e se a indicação estava ou não aprovada no registro da Anvisa, FDA e EMA. Para os casos não envolvendo indicação aprovada pela agência brasileira, foi realizada uma consulta ao relatório detalhado do caso no S-Codes de forma a identificar se havia informação sobre óbito e/ou informações adicionais sobre o quadro clínico do paciente.

Em 2017, foi implementada uma mudança no sistema S-Codes permitindo que os documentos do processo fossem escaneados e anexados de forma a facilitar a consulta pelo Grupo de Coordenação das Demandas Estratégicas em Saúde (GCodes), que fica na capital do Estado. O escaneamento e upload dos documentos no S-Codes são realizados por colaboradores em cada DRS, mas não é obrigatório, pois depende da capacidade operacional de cada local. 
Para cada demanda, foi realizada uma consulta para identificar se havia ou não documentos escaneados. Além da caracterização de todos os processos, foi realizada análise complementar de todos os processos com, pelo menos, um documento escaneado, para coletar os seguintes dados: informação sobre metástase; tratamento prévio; e teste genético para determinar presença de mutação em genes - seja por meio de resultados laboratoriais ou laudo médico.

\section{Resultados}

A tabela 1 mostra que, apesar de os seis medicamentos terem aprovação de registro nas três agências sanitárias, há diferença nas indicações aprovadas em cada uma delas. Em geral, há mais indicações aprovadas pela FDA: por exemplo, os medicamentos pembrolizumabe com 13 indicações e nivolumabe, com nove, enquanto os mesmos tiveram aprovação na Anvisa para cinco e quatro indicações, respectivamente. Também há especificações diferentes para a indicação do mesmo medicamento entre as agências, reforçando ou não a necessidade de uso prévio de outros medicamentos ou ainda o conhecimento do nível de expressão da proteína PDL1, que é um marcador que pressupõe maior chance de sucesso com o uso do medicamento, por exemplo.

Tabela 1. Indicações aprovadas no registro sanitário em três agências - Food and Drug Administration (FDA), European Medicines Agency (EMA) e Agência Nacional de Vigilância Sanitária (Anvisa) para os inibidores de checkpoints imunológicos

\begin{tabular}{|c|c|c|c|c|c|c|c|c|c|c|c|c|c|c|c|c|c|c|}
\hline \multirow[b]{2}{*}{ Indicação } & \multicolumn{3}{|c|}{ Atezolizumabe } & \multicolumn{3}{|c|}{ Avelumabe } & \multicolumn{3}{|c|}{ Durvalumabe } & \multicolumn{3}{|c|}{ Ipilimumabe } & \multicolumn{3}{|c|}{ Nivolumabe } & \multicolumn{3}{|c|}{ Pembrolizumabe } \\
\hline & FDA & EMA & Anvisa & FDA & EMA & Anvisa & FDA & EMA & Anvisa & FDA & EMA & Anvisa & FDA & EMA & Anvisa & FDA & EMA & Anvisa \\
\hline Melanoma & & & & & & & & & & $x$ & $x$ & $x$ & $x$ & $x$ & $x$ & $x$ & $x$ & $x$ \\
\hline $\begin{array}{l}\text { Câncer de } \\
\text { pulmão de } \\
\text { não- } \\
\text { pequenas } \\
\text { células }\end{array}$ & $X^{1,2}$ & $X^{2,3}$ & $X^{2,3}$ & & & & $\mathrm{X}^{*, 1}$ & $X^{1,4}$ & $X^{1}$ & & & & $X^{1,2,7}$ & $x$ & $\mathrm{X}^{1,2,7}$ & $X^{1,2,4}$ & $X^{2,3,4}$ & $X^{1,2,4}$ \\
\hline $\begin{array}{l}\text { Câncer de } \\
\text { pulmão de } \\
\text { pequenas } \\
\text { células }\end{array}$ & $x$ & & $x$ & & & & & & & & & & $X^{*, 1,3}$ & & & $X^{1,3}$ & & \\
\hline $\begin{array}{l}\text { Carcinoma } \\
\text { urotelial }\end{array}$ & $X^{\star 1,4,5}$ & $X^{1,4,5}$ & $X^{1,4,5}$ & $X^{*, 1}$ & & & $X^{1}$ & & $X^{1}$ & & & & $X^{*, 1}$ & $X^{1}$ & & $X^{* 1,4,5}$ & $X^{1,4,5}$ & $X^{1,4,5}$ \\
\hline $\begin{array}{l}\text { Câncer } \\
\text { gástrico }\end{array}$ & & & & & & & & & & & & & & & & $X^{1,3,4}$ & & $X^{1,3,4}$ \\
\hline $\begin{array}{l}\text { Linfoma de } \\
\text { Hodgkin } \\
\text { clássico }\end{array}$ & & & & & & & & & & & & & $X^{* 3}$ & $X^{3}$ & $X^{3}$ & $X^{\star, 3}$ & $\mathrm{X}^{3}$ & $\mathrm{X}^{3}$ \\
\hline
\end{tabular}




\begin{tabular}{|c|c|c|c|c|c|c|c|c|c|c|c|c|c|c|c|c|c|c|}
\hline \multirow[b]{2}{*}{ Indicação } & \multicolumn{3}{|c|}{ Atezolizumabe } & \multicolumn{3}{|c|}{ Avelumabe } & \multicolumn{3}{|c|}{ Durvalumabe } & \multicolumn{3}{|c|}{ Ipilimumabe } & \multicolumn{3}{|c|}{ Nivolumabe } & \multicolumn{3}{|c|}{ Pembrolizumabe } \\
\hline & FDA & EMA & Anvisa & FDA & EMA & Anvisa & FDA & EMA & Anvisa & FDA & EMA & Anvisa & FDA & EMA & Anvisa & FDA & EMA & Anvisa \\
\hline $\begin{array}{l}\text { Câncer de } \\
\text { células } \\
\text { escamosas } \\
\text { de cabeça e } \\
\text { pescoço }\end{array}$ & & & & & & & & & & & & & $\mathrm{X}^{1}$ & $X^{1}$ & & $X^{1,4}$ & $X^{1,4}$ & \\
\hline $\begin{array}{l}\text { Carcinoma de } \\
\text { células renais }\end{array}$ & & & & $\mathrm{X}^{*}$ & & & & & & $\mathrm{x}$ & $\mathrm{x}$ & & $\mathrm{X}^{*, 1}$ & $X^{3,6}$ & $x^{3}$ & $\mathrm{x}$ & & \\
\hline $\begin{array}{l}\text { Carcinoma de } \\
\text { células de } \\
\text { Merkel }\end{array}$ & & & & $\mathrm{x}$ & $X^{6}$ & $x^{6}$ & & & & & & & & & & $\mathrm{X}^{*}$ & & \\
\hline $\begin{array}{l}\text { Carcinoma } \\
\text { hepatocelular }\end{array}$ & & & & & & & & & & & & & $\mathrm{X}^{*}$ & & & $\mathrm{X}^{*}$ & & \\
\hline $\begin{array}{l}\text { Câncer } \\
\text { cervical }\end{array}$ & & & & & & & & & & & & & & & & $X^{\star, 3,4}$ & & \\
\hline $\begin{array}{l}\text { Câncer com } \\
\text { instabilidade } \\
\text { de } \\
\text { microssatélites } \\
\text { (alta) } \\
\end{array}$ & & & & & & & & & & & & & & & & $X^{*, 1,3}$ & & \\
\hline $\begin{array}{l}\text { Linfoma } \\
\text { primário de } \\
\text { células B do } \\
\text { mediastino }\end{array}$ & & & & & & & & & & & & & & & & $X^{*, 3}$ & & \\
\hline $\begin{array}{l}\text { Câncer } \\
\text { colorretal } \\
\end{array}$ & & & & & & & & & & $\mathrm{X}^{*}$ & & & $\mathrm{X}^{*, 1}$ & & & & & \\
\hline $\begin{array}{l}\text { Câncer de } \\
\text { mama triplo } \\
\text { negativo }\end{array}$ & $X^{\star, 4}$ & & & & & & & & & & & & & & & & & \\
\hline
\end{tabular}

Fonte: elaborado pelos autores.

1 Progressão da doença durante ou pós-tratamento prévio à base de platina.

2 Previamente tratados com medicamentos anti-EFGR ou anti-ALK.

3 Progressão da doença durante ou pós-tratamento prévio.

4 Tumor expressa PD-L1.

${ }^{5}$ Pacientes não elegíveis a tratamento à base de platina

${ }^{6}$ Somente para adultos.

${ }^{7}$ Mutação nos genes EGFR ou ALK, para serem elegíveis ao uso, pacientes devem ter progressão da doença com terapia prévia de anti-EGFR ou anti-ALK.

${ }^{*}$ Fast track: aprovação acelerada baseada em taxa da resposta tumoral e duração da resposta, sendo necessário, para a continuidade do registro, comprovação de eficácia em estudos confirmatórios.

A diferença média de tempo entre a aprovação do registro da primeira indicação nas agências dos Estados Unidos e Brasil foi de 464,5 \pm 170,8 dias, enquanto entre os Estados Unidos e a União Europeia foi de 290,0 0 176,9 dias. Na tabela 2, é possível observar tanto a diferença de tempo em dias da aprovação de registro em cada agência sanitária quanto a proporção de demandas judiciais para cada medicamento, antes e depois da aprovação na Anvisa. 
Tabela 2. Data de aprovação do registro da primeira indicação de cada inibidor de checkpoint imunológico; tempo decorrido entre o registro no Food and Drug Administration (FDA), na European Medicines Agency (EMA) e Agência Nacional de Vigilância Sanitária (Anvisa); e número de demandas antes e depois do registro no Brasil

\begin{tabular}{|c|c|c|c|c|c|c|c|c|c|}
\hline \multirow{2}{*}{ Medicamento } & \multicolumn{3}{|c|}{$\begin{array}{l}\text { Data da aprovação do } \\
\text { registro considerando a } \\
\text { primeira indicação }\end{array}$} & \multicolumn{3}{|c|}{$\begin{array}{l}\text { Diferença entre a data de } \\
\text { aprovação do registro da } \\
\text { primeira indicação } \\
\text { (tempo em dias) }\end{array}$} & \multirow{2}{*}{\begin{tabular}{|c|} 
№ e \% de \\
demandas \\
antes do \\
registro \\
da \\
primeira \\
indicação \\
no Brasil \\
\end{tabular}} & \multirow{2}{*}{\begin{tabular}{|c|} 
№ e \% de \\
demandas \\
após o \\
registro \\
da \\
primeira \\
indicação \\
no Brasil
\end{tabular}} & \multirow{2}{*}{$\begin{array}{c}\text { Total de } \\
\mathrm{n}=\mathrm{e} \% \text { de } \\
\text { demandas }\end{array}$} \\
\hline & FDA & EMA & Anvisa & $\begin{array}{c}\text { FDA } \\
\text { Anvisa }\end{array}$ & $\begin{array}{l}\text { FDA } \\
\text { EMA }\end{array}$ & $\begin{array}{c}\text { Anvisa } \\
\text { EMA }\end{array}$ & & & \\
\hline Atezolizumabe & $18 / 05 / 16$ & $20 / 09 / 17$ & $09 / 10 / 17$ & 509 & 490 & 19 & $0(0 \%)$ & $3(100 \%)$ & $3(100 \%)$ \\
\hline Avelumabe & $23 / 03 / 17$ & $18 / 09 / 17$ & $04 / 06 / 18$ & 438 & 179 & 259 & $0(0 \%)$ & $0(0 \%)$ & $0(0 \%)$ \\
\hline Durvalumabe & $01 / 05 / 17$ & $21 / 09 / 18$ & $26 / 12 / 17$ & 239 & 508 & -269 & $0(0 \%)$ & $2(100 \%)$ & $2(100 \%)$ \\
\hline Ipilimumabe & $25 / 03 / 11$ & $12 / 07 / 11$ & $11 / 06 / 12$ & 444 & 109 & 335 & $1(0 \%)$ & $61(100 \%)$ & $62(100 \%)$ \\
\hline Nivolumabe & $04 / 03 / 15$ & $20 / 07 / 15$ & $04 / 04 / 16$ & 397 & 138 & 259 & $6(3 \%)$ & $168(97 \%)$ & $\begin{array}{c}174 \\
(100 \%) \\
\end{array}$ \\
\hline Pembrolizumabe & $04 / 09 / 14$ & $17 / 07 / 15$ & $03 / 10 / 16$ & 760 & 316 & 444 & $2(5 \%)$ & $40(95 \%)$ & $42(100 \%)$ \\
\hline
\end{tabular}

Fonte: Elaboração própria a partir das informações obtidas nos websites das agências sanitárias e no sistema S-Codes

Chama a atenção que o medicamento durvalumabe foi o único que teve registro aprovado na Anvisa antes da aprovação na EMA e que a maioria das demandas judiciais ocorreram após o registro do medicamento no país para, pelo menos, uma indicação. Das 283 demandas judiciais envolvendo esses medicamentos, 278 (98\%) ocorreram após o registro da primeira indicação do medicamento na Anvisa. Somente em um dos casos houve solicitação de dois medicamentos diferentes para o mesmo paciente - ipilimumabe, em 2015, e pembrolizumabe, em 2019. Não foi identificado nenhum caso em que dois medicamentos tenham sido solicitados simultaneamente.

A tabela 3 mostra que, embora na maioria dos casos haja indicação aprovada na Anvisa para o tipo de câncer tratado, também há 14 solicitações para situações em que não há indicação em nenhuma das agências sanitárias; 8 para uso do nivolumabe no tratamento do câncer de células escamosas de cabeça e pescoço; uma indicação que não está aprovada pela Anvisa, mas é aprovada na FDA e EMA; e 5 casos com indicações aprovadas apenas pela FDA. Além disso, evidenciam-se casos em que o medicamento só tem aprovação para o tratamento de um tipo da doença, como no caso do câncer de pulmão de não pequenas células e/ou de pequenas células. 
Tabela 3. Perfil das indicações presentes nas demandas para cada medicamento: presença de, pelo menos, um documento escaneado no sistema S-Codes e indicação prevista no registro sanitário na Agência Nacional de Vigilância Sanitária (Anvisa)

\begin{tabular}{|c|c|c|c|c|}
\hline Medicamento & Doença & $\begin{array}{c}\text { № de } \\
\text { demandas }\end{array}$ & $\begin{array}{c}\text { № de } \\
\text { demandas } \\
\text { com } \\
\text { documentos } \\
\text { escaneados }\end{array}$ & Indicação Anvisa \\
\hline \multirow{3}{*}{ Atezolizumabe } & \multirow[t]{2}{*}{$\begin{array}{l}\text { Câncer de } \\
\text { pulmão }\end{array}$} & \multirow[t]{2}{*}{2} & \multirow[t]{2}{*}{$1(50 \%)$} & $\begin{array}{l}\text { Câncer de pulmão de não-pequenas células } \\
\text { (CPNPC) } \\
\text { Indicado como monoterapia para pacientes } \\
\text { adultos com CPNPC localmente avançado } \\
\text { ou metastático: } \\
\text { - após quimioterapia prévia; } \\
\text { - pacientes com mutações ativadoras EGFR } \\
\text { ou mutações tumorais ALK positivo devem } \\
\text { ter recebido terapia-alvo para essas } \\
\text { alterações anteriormente. }\end{array}$ \\
\hline & & & & $\begin{array}{l}\text { Câncer de pulmão de pequenas células } \\
(C P C P) \\
\text { Indicado em combinação com carboplatina } \\
\text { e etoposídeo, como primeira linha de } \\
\text { pacientes adultos com CPPC em estágio } \\
\text { extensivo. }\end{array}$ \\
\hline & $\begin{array}{l}\text { Carcinoma } \\
\text { urotelial }\end{array}$ & 1 & $0(0 \%)$ & $\begin{array}{l}\text { Carcinoma urotelial (CU) } \\
\text { Indicado como monoterapia para pacientes } \\
\text { com CU localmente avançado ou } \\
\text { metastático, após quimioterapia prévia à } \\
\text { base de platina; ou para pacientes que } \\
\text { sejam considerados inelegíveis à cisplatina } \\
\text { e que tenham tumores com expressão de } \\
\text { PD-L1 } \geq 5 \% \text {. }\end{array}$ \\
\hline Durvalumabe & $\begin{array}{l}\text { Câncer de } \\
\text { pulmão }\end{array}$ & 2 & $1(50 \%)$ & $\begin{array}{l}\text { Câncer de pulmão de pequenas células } \\
(C P C P) \\
\text { Indicado para o tratamento de pacientes } \\
\text { com câncer de pulmão não pequenas } \\
\text { células (CPNPC) estágio III irressecável, } \\
\text { cuja doença não progrediu após a terapia de } \\
\text { quimiorradiação à base de platina. }\end{array}$ \\
\hline \multirow[t]{4}{*}{ Ipilimumabe } & $\begin{array}{l}\text { Câncer de } \\
\text { pulmão }\end{array}$ & 3 & $1(50 \%)^{*}$ & $\begin{array}{l}\text { Câncer de pulmão não pequenas células } \\
\text { (CPNPC) } \\
\text { Indicado para o tratamento de pacientes } \\
\text { com câncer de pulmão não pequenas } \\
\text { células (CPNPC) estágio III irressecável, } \\
\text { cuja doença não progrediu após a terapia de } \\
\text { quimiorradiação à base de platina. }\end{array}$ \\
\hline & Melanoma & 56 & $9(16 \%)$ & $\begin{array}{l}\text { Indicado para o tratamento de melanoma } \\
\text { metastático ou inoperável. }\end{array}$ \\
\hline & $\begin{array}{l}\text { Neoplasia } \\
\text { maligna da } \\
\text { nasofaringe } \\
\end{array}$ & 1 & $0(0 \%)$ & \multirow[t]{2}{*}{$\begin{array}{l}\text { Não há indicação na Anvisa, nem nas } \\
\text { demais agências sanitárias. }\end{array}$} \\
\hline & Mesotelioma & 1 & $0(0 \%)$ & \\
\hline
\end{tabular}




\begin{tabular}{|c|c|c|c|c|}
\hline Medicamento & Doença & $\begin{array}{c}\text { № de } \\
\text { demandas }\end{array}$ & $\begin{array}{c}\text { № de } \\
\text { demandas } \\
\text { com } \\
\text { documentos } \\
\text { escaneados }\end{array}$ & Indicação Anvisa \\
\hline & $\begin{array}{l}\text { Neoplasia } \\
\text { maligna da } \\
\text { próstata }\end{array}$ & 1 & $0(0 \%)$ & \\
\hline \multirow{9}{*}{ Nivolumabe } & Melanoma & 83 & $33(40 \%)$ & $\begin{array}{l}\text { Indicado em monoterapia; ou em } \\
\text { combinação com ipilimumabe no tratamento } \\
\text { de melanoma avançado (irressecável ou } \\
\text { metastático); ou indicado para o tratamento } \\
\text { adjuvante de adultos com melanoma com } \\
\text { envolvimento de linfonodos ou doença } \\
\text { metastática completamente ressecada. }\end{array}$ \\
\hline & \multirow[b]{2}{*}{$\begin{array}{l}\text { Câncer de } \\
\text { pulmão }\end{array}$} & \multirow[b]{2}{*}{27} & \multirow[b]{2}{*}{$8(30 \%)$} & $\begin{array}{l}\text { Câncer de pulmão de pequenas células } \\
(C P C P) \\
\text { Não tem indicação, somente indicação pelo } \\
\text { FDA }^{4} \text {. }\end{array}$ \\
\hline & & & & $\begin{array}{l}\text { Câncer de pulmão não pequenas células } \\
\text { (CPNPC) } \\
\text { Em monoterapia, indicado para o } \\
\text { tratamento de câncer de pulmão de células } \\
\text { não pequenas (CPNPC) localmente } \\
\text { avançado ou metastático, com progressão } \\
\text { após quimioterapia à base de platina. } \\
\text { Pacientes com mutação EGFR ou ALK } \\
\text { devem ter progredido após tratamento com } \\
\text { antiEGFR e anti-ALK antes de iniciar com } \\
\text { nivolumabe. }\end{array}$ \\
\hline & $\begin{array}{l}\text { Carcinoma } \\
\text { urotelial }\end{array}$ & 25 & $12(48 \%)$ & $\begin{array}{l}\text { Indicado para o tratamento de carcinoma de } \\
\text { células renais avançado após terapia } \\
\text { antiangiogênica prévia; ou_em combinação } \\
\text { com ipilimumabe para o tratamento em } \\
\text { primeira linha de pacientes adultos com } \\
\text { carcinoma de células renais avançado ou } \\
\text { metastático, que possuem risco } \\
\text { intermediário ou alto (desfavorável). }\end{array}$ \\
\hline & $\begin{array}{l}\text { Linfoma de } \\
\text { Hodgkin }\end{array}$ & 16 & 15 (94\%) & $\begin{array}{l}\text { Linfoma de Hodgkin clássico } \\
\text { Indicado em monoterapia em pacientes em } \\
\text { recidiva ou refratário após transplante } \\
\text { autólogo de células-tronco (TACT), seguido } \\
\text { de tratamento com brentuximabe vedotina. }\end{array}$ \\
\hline & $\begin{array}{l}\text { Câncer de } \\
\text { células } \\
\text { escamosas } \\
\text { de cabeça e } \\
\text { pescoço }\end{array}$ & 8 & $2(25 \%)$ & $\begin{array}{l}\text { Não há indicação na Anvisa, somente } \\
\text { indicação pela FDA e EMA (câncer de } \\
\text { células escamosas de cabeça e pescoço) }{ }^{1} \text {. }\end{array}$ \\
\hline & $\begin{array}{l}\text { Câncer } \\
\text { colorretal }\end{array}$ & 3 & $0(0 \%)$ & $\begin{array}{l}\text { Não tem indicação, somente indicação pela } \\
\text { FDA }{ }^{2} \text {. }\end{array}$ \\
\hline & $\begin{array}{l}\text { Carcinoma de } \\
\text { células } \\
\text { hepáticas }\end{array}$ & 1 & $0(0 \%)$ & $\begin{array}{l}\text { Não tem indicação, somente indicação pela } \\
\text { FDA }^{3} \text {. }\end{array}$ \\
\hline & $\begin{array}{l}\text { Neoplasia } \\
\text { maligna dos }\end{array}$ & 1 & $0(0 \%)$ & $\begin{array}{l}\text { Não há indicação na Anvisa, nem nas } \\
\text { demais agências sanitárias. }\end{array}$ \\
\hline
\end{tabular}




\begin{tabular}{|c|c|c|c|c|}
\hline Medicamento & Doença & $\begin{array}{c}\text { № de } \\
\text { demandas }\end{array}$ & $\begin{array}{c}\text { № de } \\
\text { demandas } \\
\text { com } \\
\text { documentos } \\
\text { escaneados }\end{array}$ & Indicação Anvisa \\
\hline & $\begin{array}{l}\text { ossos e } \\
\text { cartilagens } \\
\text { articulares }\end{array}$ & & & \\
\hline & Mesotelioma & 1 & $0(0 \%)$ & \\
\hline & $\begin{array}{l}\text { Neoplasia } \\
\text { maligna da } \\
\text { mama }\end{array}$ & 1 & $0(0 \%)$ & \\
\hline & $\begin{array}{l}\text { Neoplasia } \\
\text { maligna da } \\
\text { vulva }\end{array}$ & 1 & $0(0 \%)$ & \\
\hline & $\begin{array}{l}\text { Neoplasia } \\
\text { maligna do } \\
\text { olho e anexos }\end{array}$ & 1 & $0(0 \%)$ & \\
\hline & $\begin{array}{l}\text { Neoplasia } \\
\text { maligna da } \\
\text { coróide }\end{array}$ & 1 & $0(0 \%)$ & \\
\hline & $\begin{array}{l}\text { Linfoma não- } \\
\text { Hodgkin, } \\
\text { folicular } \\
\text { (nodular) }\end{array}$ & 1 & $0(0 \%)$ & \\
\hline & $\begin{array}{l}\text { Mieloma } \\
\text { múltiplo }\end{array}$ & 1 & $0(0 \%)$ & \\
\hline & $\begin{array}{l}\text { Neoplasia } \\
\text { maligna, sem } \\
\text { especificação } \\
\text { de localização }\end{array}$ & 2 & $0(0 \%)$ & \multirow{2}{*}{$\begin{array}{l}\text { Impossível determinar com os dados } \\
\text { disponíveis. }\end{array}$} \\
\hline & $\begin{array}{l}\text { Carcinoma in } \\
\text { situ de outras } \\
\text { localizações } \\
\text { especificadas }\end{array}$ & 1 & $0(0 \%)$ & \\
\hline \multirow[b]{2}{*}{ Pembrolizumabe } & Melanoma & 25 & $16(64 \%)$ & $\begin{array}{l}\text { É indicado como monoterapia no tratamento } \\
\text { de pacientes com melanoma metastático ou } \\
\text { irressecável ou como monoterapia, no } \\
\text { tratamento adjuvante em adultos com } \\
\text { melanoma com envolvimento de linfonodos, } \\
\text { que tenham sido submetidos a ressecção } \\
\text { cirúrgica completa. }\end{array}$ \\
\hline & $\begin{array}{l}\text { Câncer de } \\
\text { pulmão }\end{array}$ & 10 & $3(30 \%)$ & $\begin{array}{l}\text { Câncer de pulmão não pequenas células } \\
\text { (CPNPC) } \\
\text { Em combinação com quimioterapia a base } \\
\text { de platina e pemetrexede para o tratamento } \\
\text { de 1a linha em pacientes com câncer de } \\
\text { pulmão de células não pequenas (CPCNP) } \\
\text { não escamoso, metastático e que não } \\
\text { possuam mutação EGFR sensibilizante ou } \\
\text { translocação ALK; ou em combinação com } \\
\text { carboplatina e paclitaxel ou paclitaxel } \\
\text { (ligado à albumina) para tratamento de } \\
\text { primeira linha em pacientes com câncer de } \\
\text { pulmão de células não pequenas (CPCNP) }\end{array}$ \\
\hline
\end{tabular}




\begin{tabular}{|c|c|c|c|c|}
\hline Medicamento & Doença & $\begin{array}{c}\text { № de } \\
\text { demandas }\end{array}$ & $\begin{array}{c}\text { № de } \\
\text { demandas } \\
\text { com } \\
\text { documentos } \\
\text { escaneados }\end{array}$ & Indicação Anvisa \\
\hline & & & & $\begin{array}{l}\text { escamoso e metastático; ou em } \\
\text { monoterapia, no tratamento de pacientes } \\
\text { com CPCNP metastático não tratado } \\
\text { anteriormente, com expressão de PD-L } 1 \geq \\
50 \% \text { e que não possuam mutação EGFR } \\
\text { sensibilizante ou translocação ALK; ou em } \\
\text { monoterapia, no tratamento de pacientes } \\
\text { com câncer de pulmão de células não } \\
\text { pequenas (CPCNP) em estado avançado, } \\
\text { com expressão PD-L1 } 1 \geq 1 \% \text {, e que tenham } \\
\text { recebido quimioterapia à base de platina. } \\
\text { Pacientes com alterações genômicas de } \\
\text { tumor EGFR ou ALK devem ter recebido } \\
\text { tratamento prévio para essas alterações } \\
\text { antes de serem tratados com } \\
\text { pembrolizumabe. } \\
\text { Câncer de pulmão de pequenas células } \\
\text { (CPPC) } \\
\text { Não tem indicação na Anvisa, apenas no } \\
\text { FDA. }\end{array}$ \\
\hline & $\begin{array}{l}\text { Carcinoma } \\
\text { urotelial }\end{array}$ & 3 & $3(100 \%)$ & $\begin{array}{l}\text { Em monoterapia no tratamento de } \\
\text { pacientes com carcinoma urotelial } \\
\text { localmente avançado ou metastático, que } \\
\text { tenham apresentado progressão da doença } \\
\text { durante ou após a quimioterapia contendo } \\
\text { platina; ou dentro de } 12 \text { meses de } \\
\text { tratamento neoadjuvante ou adjuvante com } \\
\text { quimioterapia à base de platina; ou no } \\
\text { tratamento de pacientes com carcinoma } \\
\text { urotelial localmente avançado ou } \\
\text { metastático não elegíveis à quimioterapia à } \\
\text { base de cisplatina com expressão de PD-L1 } \\
\text { (Positiva combinada) } \geq 10 \text {. }\end{array}$ \\
\hline & $\begin{array}{l}\text { Câncer } \\
\text { colorretal }\end{array}$ & 1 & $0(0 \%)$ & $\begin{array}{l}\text { Não há indicação na Anvisa, apenas no } \\
\text { FDA para câncer com instabilidade de } \\
\text { microssatélites. }\end{array}$ \\
\hline & $\begin{array}{l}\text { Neoplasia } \\
\text { maligna do } \\
\text { pâncreas }\end{array}$ & 1 & $0(0 \%)$ & \multirow{3}{*}{$\begin{array}{l}\text { Não há indicação na Anvisa, nem nas } \\
\text { demais agências sanitárias. }\end{array}$} \\
\hline & $\begin{array}{l}\text { Neoplasia } \\
\text { maligna dos } \\
\text { tecidos } \\
\text { moles do } \\
\text { retroperitônio } \\
\text { e do peritônio }\end{array}$ & 1 & $0(0 \%)$ & \\
\hline & $\begin{array}{l}\text { Neoplasia } \\
\text { maligna dos } \\
\text { grandes } \\
\text { lábios }\end{array}$ & 1 & $0(0 \%)$ & \\
\hline
\end{tabular}


Foi grande a proporção dos processos que não tinha nenhum documento digitalizado disponível no S-codes (104; 37\%); sendo frequente que não houvesse documentos escaneados para demandas envolvendo indicações não aprovadas no registro sanitário na Anvisa (tabela 3). Para 17 (63\%) dos 27 pacientes solicitando o medicamento em indicações não aprovadas pela Anvisa, havia informação no S-Codes sobre o óbito do paciente.

Somente em $13(4,5 \%)$ casos havia informação tanto sobre metástase quanto tratamento médico e teste genético. Entre os processos com documentos digitalizados, foi frequente que a gravidade do caso tenha sido destacada, caracterizando-o como metastático (78; 75\%), mas menos frequente a descrição do tratamento prévio (58; 56\%) e ainda mais escassa a informação referente a resultados de testes genéticos (26; 25\%): 5 com resultado do teste anexado ao processo, e 21 com laudo médico citando resultado. A tabela 4 mostra os resultados referentes ao perfil genético e/ou molecular do tumor.

Tabela 4. Resultados de testes genéticos e/ou moleculares identificados nos documentos escaneados disponíveis no sistema S-Codes (resultado do teste ou indicação de resultado no laudo médico)

\begin{tabular}{|c|c|c|c|}
\hline Medicamento & Indicação & Resultado & № \\
\hline \multirow{2}{*}{ Ipilimumabe } & \multirow{2}{*}{ Melanoma } & BRAF positivo & 2 \\
\hline & & EGFR negativo & 1 \\
\hline \multirow{5}{*}{ Nivolumabe } & Câncer de pulmão & EGFR negativo; ALK negativo & 1 \\
\hline & \multirow{4}{*}{ Melanoma } & BRAF negativo; CKIT negativo & 1 \\
\hline & & BRAF positivo & 9 \\
\hline & & BRAF negativo; NRAS positivo; C-KIT negativo & 1 \\
\hline & & BRAF negativo & 1 \\
\hline \multirow{7}{*}{ Pembrolizumabe } & \multirow[t]{2}{*}{ Câncer de pulmão } & $\begin{array}{l}\text { EGFR negativo; ALK negativo; expressão de PDL1 } \\
\text { positivo }\end{array}$ & 1 \\
\hline & & EGFR negativo; expressão de PDL1 (60\%) & 1 \\
\hline & \multirow{5}{*}{ Melanoma } & EGFR negativo; expressão de PDL1 (60\%) & 1 \\
\hline & & BRAF negativo & 1 \\
\hline & & BRAF negativo; CKIT negativo & 2 \\
\hline & & BRAF negativo; CKIT negativo & 1 \\
\hline & & BRAF positivo & 3 \\
\hline
\end{tabular}




\section{Discussão}

A comparação das bulas mostrou que, de forma geral, a FDA tende a ser menos rigorosa na aprovação de novas indicações para o medicamento, com muitas aprovações fast track, ou seja, aprovação acelerada baseada em taxa da resposta tumoral e duração da resposta, sendo necessário, para a continuidade do registro, comprovação de eficácia em estudos confirmatórios (17). As aprovações aceleradas têm caráter condicional, mas uma vez aprovada e se o medicamento continuar a ser prescrito, raramente é revogado o registro (18). A indicação de pembrolizumabe é um exemplo, pois, apesar da aprovação acelerada, os resultados de um estudo confirmatório não mostraram melhora dos resultados mais relevantes, como sobrevida global dos pacientes (19).

Os dados da tabela 1 e 2 mostram que o Brasil segue a FDA com frequência, com cerca de um ano e meio de diferença para o registro da primeira indicação. Apesar disso, a grande maioria das demandas foi iniciada após o registro de cada medicamento no país para ao menos uma indicação, ou seja, seriam processos que não se enquadrariam no disposto no RE n 657.718/2019. Logo, os desafios continuam para a discussão do financiamento para o uso off-label desses medicamentos pelo SUS e também do enquadramento do paciente no grupo com maior chance de resposta a esse tratamento, que é benéfico para apenas uma pequena proporção de pacientes. Considerando os seis medicamentos analisados, e de acordo com cada tipo de tumor, a estimativa de resposta aos tratamentos a partir de 2018 é de 7,09\% para câncer de pulmão de não pequenas células; 1,02\% para carcinoma urotelial; e 0,92\% para melanoma (16).

Um estudo que avaliou 68 indicações de medicamentos oncológicos aprovados pela EMA, entre 2009 e 2013, mostrou que 39 (57\%) entraram no mercado europeu sem que tenham comprovado melhora na sobrevivência ou qualidade de vida dos pacientes; nos casos em que havia comprovação estatística de melhoria, a maioria não representava benefício clínico (20). Cenário muito semelhante já havia sido demonstrado nos Estados Unidos, considerando as aprovações da $\operatorname{FDA}(21,22)$. Existe a preocupação de que a aprovação baseada em escassa evidência como última alternativa para os pacientes possa promover expectativas irreais, tanto para os prescritores quanto pacientes e familiares. Discute-se que a falta de oferta de alternativas, como cuidados paliativos, aumenta o risco de tratamento inadequado ou fútil com quimioterapia e imunoterapia (23). 
Chama a atenção ainda que o montante total gasto pelos sistemas de saúde com esses medicamentos tem aumentado e ameaça a sustentabilidade desses, ao mesmo tempo em que, na grande maioria das indicações, não há expectativa de cura da doença (20). O custo médio de um novo medicamento oral para o controle do câncer em 2014 era de 135.000 dólares por ano, o que já era seis vezes o custo de medicamentos similares aprovados no início dos anos 2000, após o ajuste da inflação; em 2017, esse valor já teria chegado a 475.000 dólares por paciente para uma terapia personalizada baseada em células para leucemia infantil $(24,25)$. Em 2018, somente com a aquisição de nivolumabe e pembrolizumabe para 86 pacientes, o gasto estimado da SES/SP foi superior a 1,6 milhão de reais - dados não publicados.

Embora quase todas as demandas tenham sido iniciadas após o registro do medicamento no país, foram identificadas situações de uso do medicamento fora da indicação aprovada na Anvisa, e casos em que não havia aprovação em nenhuma das agências sanitárias analisadas. O fato de que a maioria desses solicitantes tenha ido a óbito sugere que o uso desses medicamentos foi realizado como última alternativa para esses pacientes, o que traz novo elemento de dificuldade para a tomada de decisão.

É importante ressaltar que faltam informações muito relevantes para a análise da indicação e chance de resposta ao medicamento. Um bom exemplo é o câncer de pulmão; só foi identificado uma demanda onde o quadro clínico foi descrito especificamente como câncer de pulmão de não pequenas células, enquanto a indicação em bula é sempre especificamente relacionada ao tipo de câncer de pulmão.

O fato de que a maioria das demandas não tinha documentos escaneados no sistema S-Codes deve-se a dificuldades como falta de recursos humanos em algumas DRS e à adoção recente dessa mudança do sistema. Mesmo quando havia, pelo menos, um documento escaneado, não há como garantir que todos os documentos do processo de fato foram escaneados, o que resulta em uma limitação importante desse trabalho.

Para os casos em que havia documentos escaneados, chama a atenção que a maioria apresentava descrição do quadro como metastático, o que tanto é um indício da gravidade dos casos quanto da pertinência em relação à indicação prevista na bula desses medicamentos - da mesma forma que a descrição de tratamento prévio.

O desenvolvimento desses novos medicamentos e seus bons resultados para uma 
parcela de pacientes, ainda que pequena, são de grande importância e podem ser considerados novos marcos na história da oncologia. A possibilidade de que a aprovação do registro sanitário se baseie em alterações genômicas em comum, ao invés do sítio primário do tumor $(8,26,27)$; e a necessidade de que profissionais de saúde e do direito passem a ter que compreender o que significam essas mutações, e como isso pode influenciar/determinar a resposta ou não ao tratamento, ou seja, passem a adquirir conhecimentos em farmacogenômica, também são marcos importantes.

No caso das alterações genéticas ou moleculares apresentadas na tabela 4, são apresentados dados de presença ou ausência de mutações para as quais existe terapia alvo (mutações nos genes BRAF, EGFR, ALK, C-KIT e/ou NRAS) como a presença e nível de expressão de proteínas PDL-1, o que representaria, de forma geral, melhor prognóstico com o uso dos imunoterápicos.

De fato, têm sido discutidas barreiras à integração da farmacogenômica à prática clínica, como questões éticas, legais, sociais, de tecnologia da informação, dificuldades de reembolso/pagamento dos testes, mas principalmente questões relacionadas ao conhecimento para solicitar e interpretar os resultados desses testes (28-32). A farmacogenômica é uma nova área do conhecimento e a literatura sugere que ainda é falha a formação entre profissionais de saúde para usar esse conhecimento na prática clínica (3335). Assim, pode-se prever que haja dificuldades de interpretação também por profissionais do direito.

\section{Conclusões}

A indicação em bula indica que há estudo clínico sobre o medicamento, ainda que com dados preliminares e/ou pequeno número de pacientes, provando a eficácia e segurança mínimas para uso naquela indicação. Mesmo para indicações que constam em bula, poucos pacientes podem ser elegíveis para o uso do medicamento. Entre os pacientes elegíveis para as indicações aprovadas, alguns subgrupos podem ter melhor resposta ao medicamento, sobretudo dependendo do perfil genético, determinando ou não a expressão de determinadas proteínas, por exemplo. Para indicações não aprovadas em bula, ou aprovadas na modalidade acelerada, a evidência de eficácia e segurança é ainda mais escassa ou mesmo inexistente, como no caso do uso off-label. 
Tem havido avanços nas discussões envolvendo a judicialização da saúde, mas ainda não há definição clara sobre o uso de medicamentos fora da indicação prevista em bula, o que pode ser tão experimental quanto o uso de medicamentos sem registro no país. Essa discussão na área da oncologia agrava-se proporcionalmente ao avanço e especificidades das novas tecnologias, tanto pelo quadro clínico dos pacientes - pois, para muitos, pode ser a última tentativa de tratamento - quanto para o gestor público de saúde, que se vê compelido a custear altos valores sem a certeza de eficácia terapêutica. $O$ balizamento pelas indicações de bula não é garantidor de efetividade terapêutica; portanto, somente a identificação se há ou não o registro do medicamento no país não basta. É preciso uma análise técnica embasada em conhecimentos científicos para delimitar a chance de resposta ao tratamento solicitado judicialmente e análise ainda mais criteriosa para casos de uso offlabel.

\section{Referências}

1. Brasil. Supremo Tribunal Federal. RE 657718 [Internet]. 2019 [Acesso em 30 julho 2019]. Disponível em: https://portal.stf.jus.br/processos/detalhe.asp?incidente $=4143144$

2. Gronde T van der, Uyl-de Groot CA, Pieters T. Addressing the challenge of high-priced prescription drugs in the era of precision medicine: A systematic review of drug life cycles, therapeutic drug markets and regulatory frameworks. PLoS One [Internet]. 2017;12(8):e0182613. Disponível em: http://www.ncbi.nlm.nih.gov/pubmed/28813502

3. U.S. Food and Drug Administration. Understanding Unapproved Use of Approved Drugs "Off-Label" [Internet]. Disponível em: https://www.fda.gov/patients/learn-about-expandedaccess-and-other-treatment-options/understanding-unapproved-use-approved-drugs-label [Acesso em 28 julho 2019].

4. Wittich CM, Burkle CM, Lanier WL. Ten Common Questions (and Their Answers) About Off-label Drug Use. Mayo Clin Proc [Internet]. 2012 Oct;87(10):982-90.

5. Brasil. Agência Nacional de Vigilância Sanitária. Resolução RDC n 111, de 6 de Setembro de 2016 [Internet]. 2016. Disponível em: http:/www.in.gov.br/materia//asset_publisher/Kujrw0TZC2Mb/content/id/23530126/do1-2016-09-08-resolucao-rdc-n111-de-6-de-setembro-de-2016-23530036 [Acesso em 17 jul. 2019].

6. Shea MB, Stewart M, Van Dyke H, Ostermann L, Allen J, Sigal E. Outdated Prescription Drug Labeling. Ther Innov Regul Sci. 2018 Nov 5;52(6):771-7. 
7. Yan L, Zhang W. Precision medicine becomes reality-tumor type-agnostic therapy. Cancer Commun (London, England). 2018;38(1):6.

8. Lacombe D, Burock S, Bogaerts J, Schoeffski P, Golfinopoulos V, Stupp R. The dream and reality of histology agnostic cancer clinical trials. Mol Oncol. 2014 Sep 12;8(6):1057-63.

9. Iriart JAB. Medicina de precisão/medicina personalizada: análise crítica dos movimentos de transformação da biomedicina no início do século XXI. Cad Saude Publica. 2019 Mar $25 ; 35(3)$.

10. Senado Notícias. Especialistas defendem testes genéticos para tratamento do câncer no SUS [Internet]. 2018 [Acesso em 28 jul. 2019]. Disponível em: https://www12.senado.leg.br/noticias/materias/2018/11/06/especialistas-defendem-testesgeneticos-para-tratamento-do-cancer-no-sus

11. Chang MT, Bhattarai TS, Schram AM, Bielski CM, Donoghue MTA, Jonsson $P$, et al. Accelerating Discovery of Functional Mutant Alleles in Cancer. Cancer Discov. 2018 Feb;8(2):174-83. Disponível em: http://cancerdiscovery.aacrjournals.org/lookup/doi/ 10.1158/2159-8290.CD-17-0321

2. Buljan M, Blattmann $P$, Aebersold R, Boutros M. Systematic characterization of pan-cancer mutation clusters. Mol Syst Biol. 2018 Mar 23;14(3): e7974.

3. Bartels S, Schipper E, Hasemeier B, Kreipe H, Lehmann U. Hotspot mutations in cancer genes may be missed in routine diagnostics due to neighbouring sequence variants. Exp Mol Pathol. 2018 Aug;105(1):37-40.

4. Chang MT, Asthana S, Gao SP, Lee BH, Chapman JS, Kandoth C, et al. Identifying recurrent mutations in cancer reveals widespread lineage diversity and mutational specificity. Nat Biotechnol. 2016 Feb;34(2):155-63.

5. Marquart J, Chen EY, Prasad V. Estimation of the Percentage of US Patients With Cancer Who Benefit From Genome-Driven Oncology. JAMA Oncol. 2018 Aug 1;4(8):1093.

6. Haslam A, Prasad V. Estimation of the Percentage of US Patients With Cancer Who Are Eligible for and Respond to Checkpoint Inhibitor Immunotherapy Drugs. JAMA Netw open. 2019 May 3;2(5):e192535.

7. U.S. Food and Drug Administration. Fast Track [Internet]. [Acesso em 23 julho 2019]. Disponível em: https://www.fda.gov/patients/fast-track-breakthrough-therapy-acceleratedapproval-priority-review/fast-track

8. US Government Accountability Office. New drug approval: FDA needs to enhance its oversight of drugs approved on the basis of surrogate endpoints. 
9. Healio Immuno-Oncology Resource Center. Phase 3 trial of pembrolizumab for advanced HCC fails to meet primary endpoints [Internet]. [Acesso em 02 Agosto 2019]. Disponível em: https://www.healio.com/hematology-oncology/gastrointestinalcancer/news/online/\%7Ba1a112d0-3f4c-425b-bb25-0823c56e56e6\%7D/phase-3-trial-ofpembrolizumab-for-advanced-hcc-fails-to-meet-primary-endpoints

10. Davis C, Naci H, Gurpinar E, Poplavska E, Pinto A, Aggarwal A. Availability of evidence of benefits on overall survival and quality of life of cancer drugs approved by European Medicines Agency: retrospective cohort study of drug approvals 2009-13. BMJ. 2017 Oct $4 ; j 4530$.

11. Pease AM, Krumholz HM, Downing NS, Aminawung JA, Shah ND, Ross JS. Postapproval studies of drugs initially approved by the FDA on the basis of limited evidence: systematic review. BMJ. 2017 May 3;j1680.

12. Kim C, Prasad V. Cancer Drugs Approved on the Basis of a Surrogate End Point and Subsequent Overall Survival. JAMA Intern Med. 2015 Dec 1;175(12):1992. Disponível em: http://archinte.jamanetwork.com/article.aspx?doi=10.1001/jamainternmed.2015.5868

13. Cohen D. Cancer drugs: high price, uncertain value. BMJ. 2017 Oct 4;j4543.

14. Dusetzina SB. Drug Pricing Trends for Orally Administered Anticancer Medications Reimbursed by Commercial Health Plans, 2000-2014. JAMA Oncol. 2016 Jul 1;2(7):960-1.

15. Dolgin E. Bringing down the cost of cancer treatment. Nature. 2018 Mar 8;555(7695):S26-9.

16. Le Tourneau C, Kamal M, Bièche I. Precision medicine in oncology: what is it exactly and where are we? Per Med. 2018 Sep;15(5):351-3.

17. Le Tourneau C, Borcoman E, Kamal M. Molecular profiling in precision medicine oncology. Nat Med. 2019;25(5):711-2.

18. Rodrigues-Soares F, Suarez-Kurtz G. Pharmacogenomics research and clinical implementation in Brazil. Basic Clin Pharmacol Toxicol. 2019 May 24;124(5):538-49.

19. McCarthy JJ, McLeod HL, Ginsburg GS. Genomic medicine: a decade of successes, challenges, and opportunities. Sci Transl Med. 2013 Jun 12;5(189):189sr4.

20. Klein ME, Parvez MM, Shin J-G. Clinical Implementation of Pharmacogenomics for Personalized Precision Medicine: Barriers and Solutions. J Pharm Sci. 2017 Sep;106(9):2368-79 .

21. Owusu Obeng A, Fei K, Levy K, Elsey A, Pollin T, Ramirez A, et al. Physician-Reported Benefits and Barriers to Clinical Implementation of Genomic Medicine: A Multi-Site IGNITENetwork Survey. J Pers Med. 2018 Jul 24;8(3):24. 
22. Albassam A, Alshammari S, Ouda G, Koshy S, Awad A. Knowledge, perceptions and confidence of physicians and pharmacists towards pharmacogenetics practice in Kuwait. Rovers J, editor. PLoS One . 2018 Sep 5;13(9):e0203033.

23. Stanek EJ, Sanders CL, Taber KAJ, Khalid M, Patel A, Verbrugge RR, et al. Adoption of Pharmacogenomic Testing by US Physicians: Results of a Nationwide Survey. Clin Pharmacol Ther. 2012 Mar 25;91(3):450-8.

24. Nickola TJ, Green JS, Harralson AF, O'Brien TJ. The current and future state of pharmacogenomics medical education in the USA. Pharmacogenomics. 2012 Sep;13(12):1419-25.

25. Daly AK. Is There a Need to Teach Pharmacogenetics? Clin Pharmacol Ther. 2014 Mar;95(3):245-7.

Como citar esse artigo:

Oliveira DB, Silva RAM, de Paula EA, Cassamassimo RP, Siqueira PSF, Melo DO. A judicialização de medicamentos imunoterápicos sem registro na Anvisa: o caso do Estado de São Paulo. Cadernos Ibero-Americanos de Direito Sanitário. 2019 jul./set.; 8(3): 27-47.

http://dx.doi.org/10.17566/ciads.v8i3.571 